\title{
Socioeconomic Correlates of Sedentary Behavior in Adolescents: Systematic Review and Meta-Analysis
}

\author{
Gregore I. Mielke ${ }^{1,2} \cdot$ Wendy J. Brown ${ }^{2}$ Bruno P. Nunes ${ }^{1,3}$ - Inacio C. M. Silva ${ }^{1}$. \\ Pedro C. Hallal ${ }^{1}$
}

Published online: 3 June 2016

(C) The Author(s) 2016. This article is published with open access at Springerlink.com

\begin{abstract}
Background The body of evidence on associations between socioeconomic status (SES) and sedentary behaviors in adolescents is growing.

Objectives The overall aims of our study were to conduct a systematic review and meta-analysis of this evidence and to assess whether (1) the associations between SES and sedentary behavior are consistent in adolescents from lowmiddle-income and from high-income countries, (2) the associations vary by domain of sedentary behavior, and (3) the associations vary by SES measure.

Methods We performed a systematic literature search to identify population-based studies that investigated the association between SES and sedentary behavior in adolescents (aged 10-19 years). Only studies that presented risk estimates were included. We conducted meta-analyses using random effects and univariate meta-regression and calculated pooled effect sizes (ES).
\end{abstract}

Electronic supplementary material The online version of this article (doi:10.1007/s40279-016-0555-4) contains supplementary material, which is available to authorized users.

Gregore I. Mielke

gregore.mielke@gmail.com

1 Postgraduate Program in Epidemiology, Federal University of Pelotas, Rua Marechal Deodoro 1160, 3rd floor, Pelotas 96020-220, Brazil

2 School of Human Movement and Nutrition Sciences, University of Queensland, Brisbane, QLD, Australia

3 Department of Nursing, Federal University of Pelotas, Pelotas, Brazil
Results Data from 39 studies were included; this provided 106 independent estimates for meta-analyses. Overall, there was an inverse association between SES and sedentary behavior (ES $0.89 ; 95 \%$ confidence interval [CI] 0.81-0.98). However, the direction of the association varied: in high-income countries, SES was inversely associated with sedentary behavior (ES 0.67; $95 \%$ CI 0.62-0.73), whereas in low-middle-income countries, there was a positive association between SES and sedentary behavior (ES 1.18; $95 \%$ CI 1.04-1.34). In high-income countries, the associations were strongest for screen time (ES 0.68; $95 \%$ CI 0.62-0.74) and television (TV) time (ES $0.58 ; 95 \%$ CI $0.49-0.69$ ), whereas in low-middle-income countries, the associations were strongest for 'other' screen time (i.e., computer, video, study time, but not including TV time) (ES 1.38; $95 \%$ CI 1.07-1.79). All indicators of SES were negatively associated with sedentary behavior in high-income countries, but only resources (income and assets indexes) showed a significant positive association in low-middle-income countries.

Conclusion The associations between SES and sedentary behavior are different in high- and low-middle-income countries, and vary by domain of sedentary behavior. These findings suggest that different approaches may be required when developing intervention strategies for reducing sedentary behavior in adolescents in different parts of the world. 


\section{Key Points}

Associations between socioeconomic status (SES) and sedentary behavior differ between adolescents from high- and low-middle-income countries and vary by domain of sedentary behavior.

In high-income countries, there was a strong and consistent inverse association between SES and total screen time and television (TV) time. In contrast, in low-middle-income countries, SES was not associated with total screen or TV time, but there was a positive association between SES and "other screen time" (i.e., video, computer games, or study time, but not including TV time).

This review suggests the use of different approaches in low-middle- and high-income countries for reducing sedentary behavior in adolescents.

\section{Introduction}

During the last decade, the concept of being 'sedentary' has changed. Whereas it was once understood as not meeting the guidelines for moderate to vigorous physical activity [1], the term 'sedentary behavior' is now used to describe waking behaviors that involve sitting or lying down [2]. Although the independent effects of sedentary behavior and physical activity in terms of health consequences are debated, there is consensus that the correlates of these behaviors may be different, in both adolescents and adults [3, 4].

Three recent reviews have shown that socioeconomic status (SES) is an important correlate of sedentary behavior, and that children and adolescents from lower socioeconomic backgrounds have higher levels of sedentary behavior, in both screen-based and non-screen-based activities [4-6]. In contrast, a systematic review of the correlates of sedentary behavior among school-aged children in Sub-Saharan Africa found that higher SES was associated with more sedentary behavior [7].

As correlates of different domains of sedentary behavior (such as television [TV] time, screen time, studying, etc.) are likely to differ, some studies focused on only one sedentary behavior domain [6]. However, others grouped time spent in different domains [4, 7] making it difficult to assess domain-specific correlates. Moreover, many studies focused on only one indicator of SES: either parental income, occupation, or education [8-12]. However, it is possible that, although SES measures are strongly correlated, they might influence health behavior differently [13, 14]. For example, while resources (e.g., income or assets index) might be strongly related to ownership of electronic devices at home (thereby allowing access to "screen" devices), parental education might be associated with parental rules limiting access to these devices $[15,16]$.

One limitation of much of the research to date is that most studies have focused on both children and adolescents $[5,6]$. However, the correlates of sedentary behavior may differ in children and adolescents (defined by the World Health Organization as aged 10-19 years) [17], because of increasing autonomy for decision making as young people move through their teenage years. To inform the development of effective interventions for reducing sedentary behaviors in adolescents, it is important to understand the socioeconomic determinants of the different domains of sedentary behavior at this specific life stage.

The aims of this review were, via meta-analysis, to examine the SES correlates of sedentary behavior in adolescents, and to examine whether (1) the associations between SES and sedentary behaviors are consistent in adolescents from low-middle-income and from high-income countries, (2) the associations vary by domain of sedentary behavior, and (3) the associations vary by SES measure.

\section{Methods}

\subsection{Search Strategy}

In 2015, we conducted a systematic search in the Academic Search Premier, CINAHL, Cochrane, PubMed, Scopus, SocIndex, SPORTDiscus, and Web of Science databases to identify relevant studies on associations between SES and sedentary behavior in adolescents. Groups of thesaurus terms and free terms were searched using a Boolean strategy: terms for adolescents ("adolescence" OR "adolescent" OR "adolescents" OR "teen" OR "teenager" OR "teenagers" OR “teens" OR “youth" OR “youths”) were used in AND combination with terms for sedentary behavior ("Sedentary behavior" OR "Sedentary behaviour" OR "Sedentary time" OR "Sitting time" OR "Television" OR "Screen-based" OR “TV viewing” OR "Computer use") AND terms for socioeconomic status ("Schooling attainment" OR "Family income" OR "income" OR "Socioeconomic position "OR "Socioeconomic level" OR "Economic level" OR "Assets index" OR "Poverty" OR "Deprivation" OR "Schooling” OR "education" OR "disparity" OR "ethnic" OR “inequality" OR "inequity" OR "race”). All studies published up to 19 March 2015 were considered. 


\subsection{Eligibility and Exclusion Criteria}

We considered only full-text, peer-reviewed populationbased studies focusing on adolescents (mean age 10-19 years) [17], with a measure of SES as the exposure, and a measure of sedentary behavior as the outcome, and reporting an association between SES and sedentary behavior variables. Measures of parental education, income, assets index/deprivation, and occupation were considered as indicators of SES. The search was restricted to studies published in English, Portuguese, or Spanish. Review papers, theses, and dissertations were not included.

We applied the following exclusion criteria:

(i) sedentary behavior was inappropriately defined, i.e., defined as not meeting physical activity guidelines;

(ii) the focus was on a specific clinical population (e.g., overweight or obese, people with Down syndrome or other disability; people with a specific illness);

(iii) there was no heterogeneity in socioeconomic level, i.e., only those in a specific socioeconomic level were included;

(iv) the study was an intervention that aimed to reduce sedentary behavior (with the exception of studies reporting on baseline data from intervention studies);

(v) sedentary time was an exposure instead of an outcome measure;

(vi) the study included children, adolescents, and adults, but did not present separate analyses for adolescents. In studies that included children and adolescents, but did not present separate analyses for adolescents, studies were excluded if the average age was $<10$ or $>19$ years (or where the majority of participants were not aged between 10 and 19 years);

(vii) the study did not provide data on the association between SES and sedentary behavior, from analyses of primary or secondary data (or did not provide data to enable calculation of these estimates, for example, from $2 \times 2$ tables).

\subsection{Data Extraction}

The first author (GIM) conducted the search; two independent reviewers (GIM and BPN) evaluated all abstracts. If the two reviewers were unsure, they sought consensus from all authors. Three independent reviewers (GIM, BPN, and ICMS) extracted information from all the included papers, and any disagreement was resolved by consensus in consultation with the other authors (WJB and PCH). Extracted information included authors, year of publication, country in which the study was conducted, survey year, study design, sample size, age range, type(s) of SES measures, number of SES categories, and sedentary behavior domains and definitions.

Where reported, odds ratio (OR) and respective standard errors or $95 \%$ confidence intervals (CIs) were extracted. If these data were not reported or could not be calculated, we contacted the first author of the study via email. If the authors could not be contacted, or could not supply the data, we excluded the study. We also excluded studies that only presented sedentary behavior as a continuous variable, and did not report a categorical variable for "high" sedentary behavior.

To prevent duplication, if multiple publications were available from the same data source/study population, we used the most recent or most complete data. In cases where publications had complementary information (i.e., one provided data about one sedentary domain and/or SES measure and another provided data about other associations) we included both studies. If studies reported findings for boys and girls separately, we included two independent estimates in the meta-analysis. If studies measured sedentary behavior separately on weekdays and weekends, we only used estimates from weekdays.

The manuscript was modelled on the Preferred Reporting Items for Systematic Reviews and Meta-Analyses (PRISMA) Statement [18].

\subsection{Data Management and Statistical Analysis}

Initially, we performed a general meta-analysis, with pooling of all estimates, using the original sedentary behavior domains (study, TV, video games, personal computer, screen time, or combinations of these) and SES measures (paternal, maternal, or parental education; assets index/deprivation; income; paternal, maternal, or parental occupation). We then performed a series of univariate metaregressions to investigate the main sources of heterogeneity in the overall meta-analysis (see Electronic Supplementary Material [ESM] Table S1). For these analyses, SES measures were categorised as (1) education (paternal, maternal or parental education); (2) resources (including income, assets index, and deprivation); or (3) occupation (paternal, maternal, or parental education). Sedentary behaviors were categorised as (1) screen-based (for studies that considered TV time and/or video game time and/or computer time together; (2) TV-viewing time (for studies that measured only TV time); or (3) "other" (for studies that measured computer and/or video game time and/or time spent studying, but not TV time). Data were stratified by the World Bank's country classification (low- or middle-income country; or high-income country). We used random-effects models to calculate pooled effects sizes (ES) and assessed heterogeneity using the I-squared test. 
In all the analyses, we used the lowest SES category as the reference group. Thus, effect measures higher than 1.00 indicate more sedentary behavior, and effect measures lower than 1.00 indicate less sedentary behavior in higher SES groups than in the reference (low) SES group. Comparisons of the highest and the lowest socioeconomic groups reported in each paper were included in the metaanalysis.

We conducted several sensitivity analyses to assess the robustness of the data and to explore potential sources of heterogeneity. These analyses included (1) data from studies that reported only one SES variable; (2) a comparison of studies that used two and those that used more than two SES categories; (3) exclusion of studies in which the reference category was changed to allow inclusion of the data; (4) exclusion of studies that included participants who were aged $<10$ or $>19$ years; (5) exploration of bias due to different definitions of "high" sedentary behavior, and (6) analysis of data from studies that did not stratify by sex. We used funnel plots and Egger tests to investigate publication bias.

\section{Results}

Figure 1 provides an overview of the search process. We identified 6174 references, 612 of which were identified as potentially relevant after exclusion of duplicates and those that did not meet the inclusion criteria. Of these, 444 were excluded after abstract review, mostly because they did not report an association between SES and sedentary behavior. After full review of the remaining 168 papers, 39 were considered for inclusion in the meta-analysis. These papers included 106 separate estimates of SES-sedentary time associations.

Table 1 shows the characteristics of the included studies, which were from 15 different countries: Brazil $(=12)$, the USA (=8), Australia (=4), China (=3), England (=3), and Norway $(=2)$ contributed more than one study, and the remaining nine countries contributed one each. All were conducted between 1994 and 2011 and published between 2000 and 2015; most were of cross-sectional design. Sample size ranged from small studies of $<500$ adolescents in Australia and France to large studies of $>50,000$ participants in Brazil and the USA. The age range was from 6 to 19 years, with average age between 10 and 19 in all studies. The 39 papers included nine measures of SES, including paternal, maternal, or parental education (25 studies), resources (23 studies), and parental occupation (five studies). The majority $(n=23)$ considered only one measure of SES, but five studies included three or more measures of SES. The majority of studies $(n=34)$ also used a single measure of sedentary behavior; this was most commonly TV time $(n=17)$ or a composite measure of time watching TV and playing video games or using a computer $(n=10)$. Three studies measured video game and computer time separately, and 15 measured total screen time. Most studies $(n=32)$ presented analyses for boys and girls combined; only seven presented separate analyses for boys and girls (Table 1).

The results of the overall meta-analysis (Fig. 2) showed the odds of high sedentary behavior were $11 \%$ lower in the highest SES groups than in the lowest SES groups (ES $0.89 ; 95 \%$ CI 0.81-0.98). As expected, there was substantial heterogeneity among studies $\left(I^{2}=94.8 \%\right)$; the sources of this are described below.

Table 2 presents the results of the meta-regression analyses of heterogeneity sources. The top panel of Table 2 shows the main source of heterogeneity $\left(R^{2}=37.3\right)$ was country income status; there was a negative association between SES and sedentary behavior in adolescents from high-income countries (ES 0.67; $95 \%$ CI 0.62-0.73) and a positive association in studies from low-middle-income countries (ES 1.18; $95 \%$ CI 1.04-1.30). There was also some heterogeneity $\left(R^{2}=20.2\right)$ in terms of the domain of sedentary behavior, with negative associations for screen and TV time and a positive association for "other" screen time. Heterogeneity due to the SES measure was low $\left(R^{2}=6.9\right)$.

When the data were stratified by country income (middle and lower panels of Table 2), the analyses showed that sedentary behavior domains explained $29 \%$ of the heterogeneity in high-income countries, but only $4.3 \%$ in low-middle-income countries. The association between SES and high screen and TV time was negative in highincome countries, indicating lower odds of high sedentary behavior in the highest than in the lowest SES groups. In low-middle-income countries, only the association between SES and "other" screen time was significant; there was a positive relationship, indicating greater likelihood of high "other" screen time in high SES than in low SES groups.

The way SES was measured was more important in lowmiddle-income countries $\left(R^{2}=10.8\right)$ than in high-income countries $\left(R^{2}=-3.3 \%\right)$. Among low-middle-income countries, resource measures were more strongly associated with sedentary behaviors than measures related to parental education $\left(\mathrm{OR}_{\text {meta-regression }} 1.38 ; 95 \%\right.$ CI 1.07-1.78). This pattern was not observed in high-income

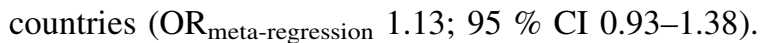

Meta-analyses showing the associations between SES and each of three sedentary behavior measures, stratified by country income, are shown in Figs. 3, 4, and 5. Data from studies that combined TV, video, and computer game time showed a strong negative association between SES and high screen time in high-income countries (ES 0.68; $95 \%$ CI 0.62-0.74) (Fig. 3). Of 30 individual estimates, half 
Fig. 1 Flowchart reporting the process for selection of papers for inclusion in the metaanalysis. $S B$ sedentary behavior, SES socioeconomic status

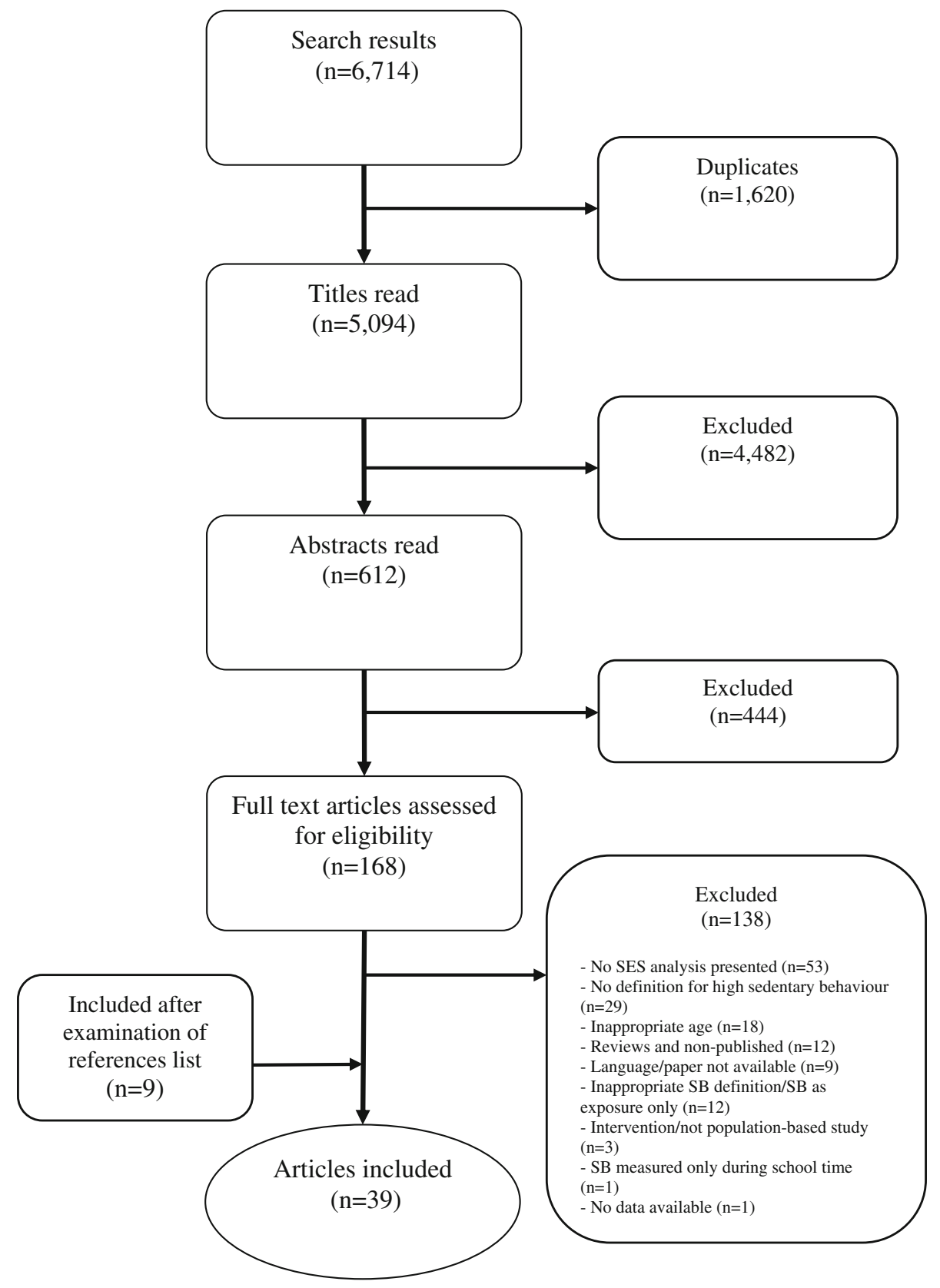

were significantly associated with lower SES and only one OR was greater than 1.00 (but not statistically significant). The same pattern was not observed among low-middleincome countries, where there was no association between SES and screen time (ES 1.06; $95 \%$ CI 0.76-1.47). Heterogeneity among estimates of SES and screen time was higher in the studies from low-middle-income countries, with some differences in the direction of the association in studies from Brazil (positive) compared with those from China and Thailand (negative) (Fig. 3).

A similar pattern of association was found when only estimates of TV viewing time were examined (Fig. 4). There was a clear inverse association between SES and TV time in high-income countries (ES 0.58; $95 \%$ CI 0.49-0.69), but no association between SES and TV time in low-middle-income countries (ES 1.08; $95 \%$ CI 0.97-1.20). This latter finding reflected the greater heterogeneity in studies from low-middle-income countries.

The meta-analysis of data from studies that included a range of sedentary domains, but not including TV time ("other" sedentary time), is shown in Fig. 5. In contrast with the findings for screen and TV-viewing time, this analysis showed that adolescents with higher SES tended to spend more time in sedentary behavior than those with lower SES, irrespective of country income status. 


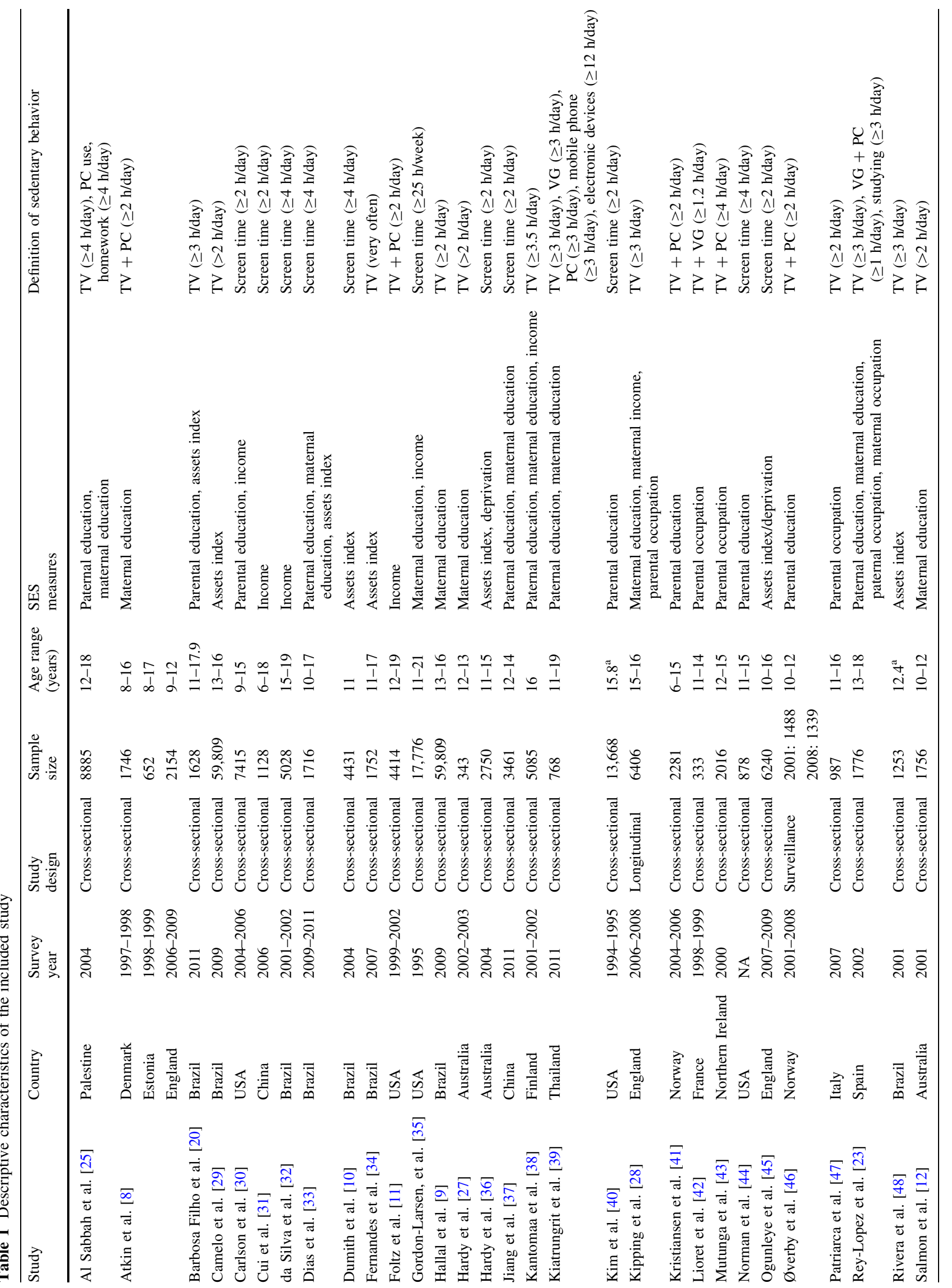




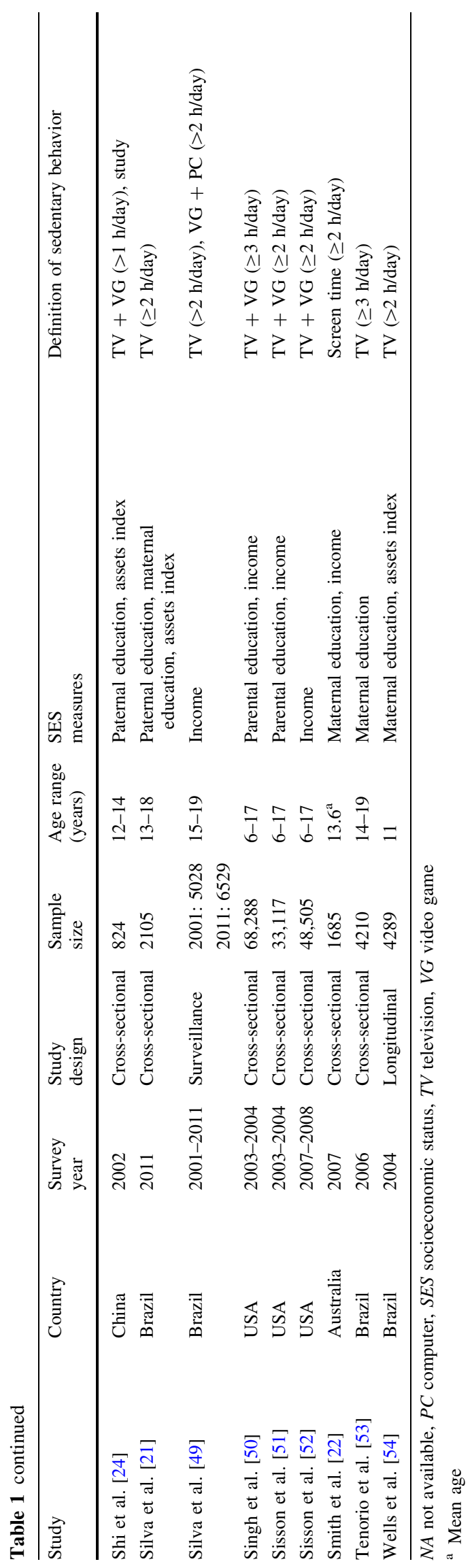

However, heterogeneity was high in the low-middle-income country studies, largely because of some very strong effects reported by one Brazilian study (Fig. 5).

A series of sensitivity analyses did not substantially change these results. Although the number of SES categories was not an important source of heterogeneity in the general meta-analysis (adjusted $R^{2}=-0.56 \%$ ), when the data were stratified by country income, there was a positive association between the number of SES categories included in the pooled-effect model and estimate of meta-analysis, but only in low-middle-income countries $\left(\mathrm{OR}_{\text {meta-regression }}\right.$ $1.21 ; 95 \%$ CI 1.02-1.44). The funnel plots and Egger's tests showed no evidence of publication bias for the studies from either low-middle-income $(p=0.309)$ or high-income countries $(p=0.179)$. Influence analyses did not show important changes to the pooled-effect sizes due to any individual study in low-middle-income countries or in high-income countries.

\section{Discussion}

Although several systematic reviews have already been conducted, this study was the first to quantify the associations between SES and sedentary behaviors in adolescents via meta-analysis techniques. By including data from more than 350,000 participants, we were able to calculate an overall pooled effect and examine the factors contributing to variations in the strength and direction of this association. Understanding the sources of variation in studies of adolescents is important, as it allows strategies for behavior change to be specifically targeted to this life stage. Overall, the pooled results from 39 studies showed that the odds of high sedentary behavior were $11 \%$ lower in the highest SES groups than in the lowest SES groups. However, substantial heterogeneity existed, with contrasting findings in studies from high- and low-middle-income countries, for different domains of sedentary behavior, and - to a lesser extent-by the SES variable used.

There was a negative association between SES and sedentary behavior in adolescents from high-income countries (hence adolescents from low SES backgrounds were more likely to have high levels of sedentary behavior than their high-SES counterparts). The reverse was true in low-middle-income countries, where the higher SES adolescents were more likely to be highly sedentary. This contrast was underpinned by differences in domain-specific associations, with inverse associations between SES and screen and TV time in high-income countries and a positive association between SES and 'other' screen time in lowmiddle-income countries.

We found the associations between SES and sedentary behavior varied in the different country income groups, but 
Fig. 2 General meta-analysis of the association between socioeconomic status and sedentary behavior. $A I$ assets index, $C I$ confidence interval, $E S$ effect size, $F E$ paternal education, $F O$ paternal occupation, $I$ income, $M E$ maternal education, $M O$ maternal occupation, $P C$ computer, $P E$ parental education, $P O$ parental occupation, $S B$ sedentary behavior, $S E S$ socioeconomic status, $T V$ television, $V G$ video game. $T V+$ indicates estimates based on studies that measure sedentary behavior as: a $\mathrm{TV}+\mathrm{VG}$ or $\mathbf{b} \mathrm{TV}+\mathrm{PC}$

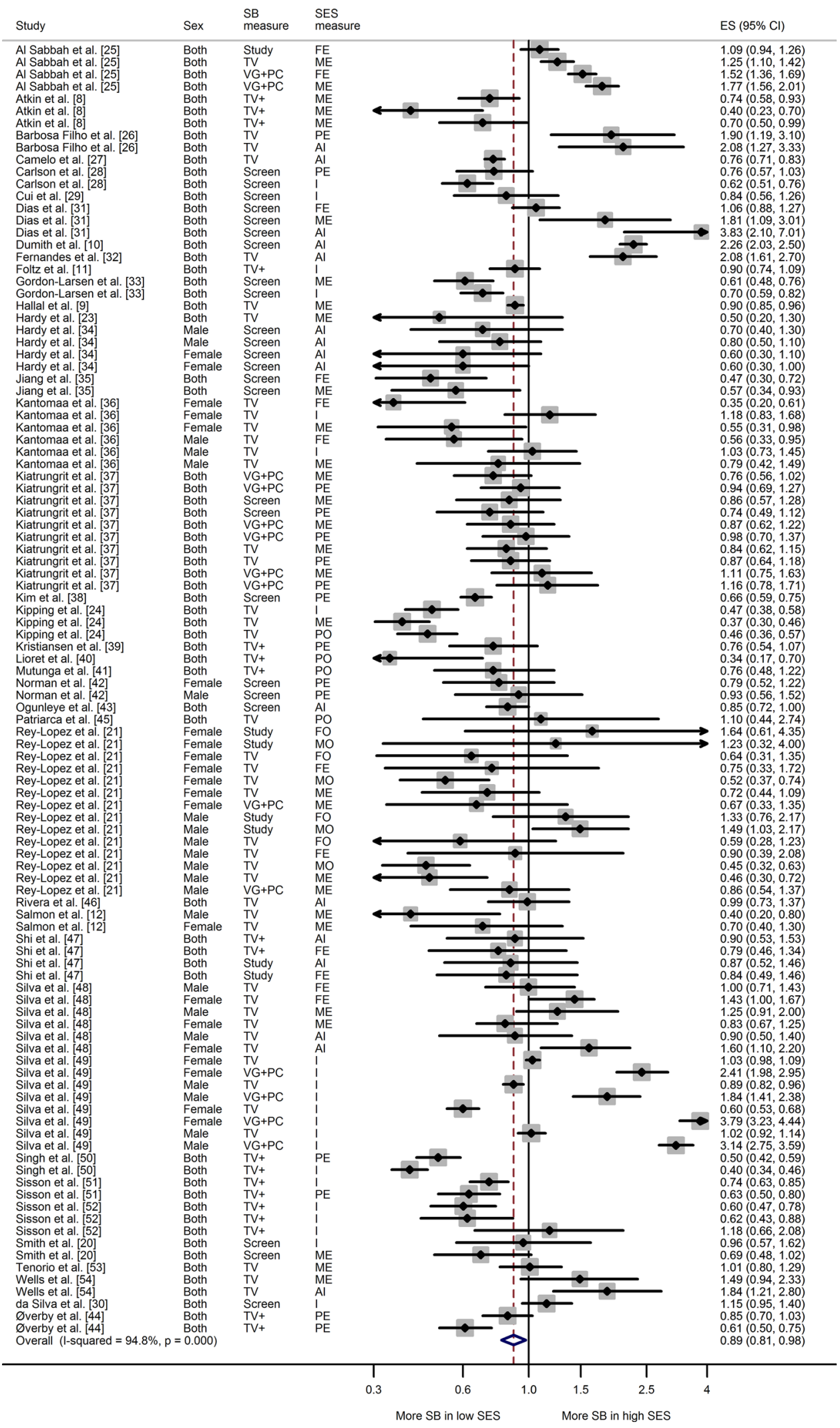


Table 2 General meta-analysis showing heterogeneity sources, and meta-regression of the associations between socioeconomic status and high sedentary behavior variables in low-middle-income and high-income countries (106 estimates from 39 studies)

\begin{tabular}{|c|c|c|c|c|c|}
\hline Variables & $n^{\mathrm{a}}$ & ES pooled $(95 \% \mathrm{CI})$ & $I^{2}$ & $\begin{array}{l}\text { Meta-regression } \\
\text { OR }(95 \% \mathrm{CI})\end{array}$ & $\begin{array}{l}\% \text { Heterogeneity } \\
\text { explained }\left(R^{2}\right)\end{array}$ \\
\hline \multicolumn{6}{|l|}{ General } \\
\hline \multicolumn{6}{|c|}{ Sedentary behavior definition } \\
\hline Screen time $\mathrm{e}^{\mathrm{b}}$ & 42 & $0.77(0.66-0.90)$ & 93.6 & Index & \multirow[t]{3}{*}{20.2} \\
\hline $\mathrm{TV}$ & 43 & $0.86(0.77-0.95)$ & 90.0 & $1.11(0.91-1.35)$ & \\
\hline Other $^{\mathrm{c}}$ & 21 & $1.32(1.06-1.66)$ & 94.0 & $1.74(1.37-2.21)$ & \\
\hline \multicolumn{6}{|l|}{ SES } \\
\hline Education & 38 & $0.82(0.74-0.91)$ & 89.3 & Index & \multirow[t]{3}{*}{6.9} \\
\hline Resource & 56 & $1.06(0.89-1.26)$ & 97.3 & $1.30(1.06-1.58)$ & \\
\hline Occupation & 12 & $0.73(0.53-1.00)$ & 78.1 & $0.89(0.64-1.25)$ & \\
\hline \multicolumn{6}{|l|}{ Country income ${ }^{\mathrm{d}}$} \\
\hline Low-middle income & 49 & $1.18(1.04-1.34)$ & 96.0 & Index & \multirow[t]{2}{*}{37.3} \\
\hline High-income & 57 & $0.67(0.62-0.73)$ & 74.9 & $0.57(0.49-0.67)$ & \\
\hline \multicolumn{6}{|c|}{ Low-middle-income countries } \\
\hline \multicolumn{6}{|c|}{ Sedentary behavior definition } \\
\hline Screen time ${ }^{\mathrm{b}}$ & 12 & $1.06(0.76-1.47)$ & 93.5 & Index & \multirow[t]{3}{*}{4.3} \\
\hline $\mathrm{TV}$ & 22 & $1.08(0.97-1.20)$ & 89.8 & $1.06(0.75-1.48)$ & \\
\hline Other $^{\mathrm{c}}$ & 15 & $1.38(1.07-1.79)$ & 95.6 & $1.31(0.91-1.89)$ & \\
\hline \multicolumn{6}{|l|}{ SES } \\
\hline Education & 28 & $1.04(0.92-1.17)$ & 86.2 & Index & \multirow[t]{3}{*}{10.8} \\
\hline Resource & 21 & $1.42(1.13-1.79)$ & 98.0 & $1.38(1.07-1.78)$ & \\
\hline Occupation & 0 & - & - & - & \\
\hline \multicolumn{6}{|l|}{ High-income countries } \\
\hline \multicolumn{6}{|c|}{ Sedentary behavior definition } \\
\hline Screen time ${ }^{\mathrm{b}}$ & 30 & $0.68(0.62-0.74)$ & 73.8 & Index & \multirow[t]{3}{*}{28.9} \\
\hline $\mathrm{TV}$ & 21 & $0.58(0.49-0.69)$ & 67.0 & $0.85(0.71-1.02)$ & \\
\hline Other $^{c}$ & 6 & $1.15(0.87-1.52)$ & 22.7 & $1.69(1.22-2.34)$ & \\
\hline \multicolumn{6}{|l|}{ SES } \\
\hline Education & 28 & $0.63(0.57-0.70)$ & 57.8 & Index & \multirow[t]{3}{*}{-3.3} \\
\hline Resource & 17 & $0.72(0.61-0.84)$ & 85.0 & $1.13(0.93-1.38)$ & \\
\hline Occupation & 12 & $0.73(0.53-1.00)$ & 78.1 & $1.11(0.86-1.44)$ & \\
\hline Total & 106 & $0.89(0.81-0.98)$ & 94.8 & - & - \\
\hline
\end{tabular}

$C I$ confidence interval, ES effect size, $O R$ odds ratio, SES socioeconomic status

${ }^{a}$ Represents the number of estimates available

${ }^{\mathrm{b}}$ Estimates based on studies that measured sedentary behavior as time spent in TV + computer + video games + other screen-based activities

${ }^{c}$ Estimates based on studies that measured sedentary behavior as time spent in computer, video game, study time, but not including TV time

d According to World Bank classification

this variation was underpinned by complex inter-relationships with both domains of sedentary behavior and measures of SES. Differences in access to TVs and computer games in high- and low-middle-income households may explain this finding, as studies in high-income countries have consistently found that adolescents from homes with more TVs and computers, and those with a TV in the bedroom, report more screen-based sedentary behavior [6]. However, access to TVs and video/computer games differs in low-middle-income countries. For example, between
2000 and 2012, the proportion of households in Brazil with a TV increased from 87 to $95 \%$, while the proportion with a computer increased from 10 to $39 \%$. These trends were strongly related to economic status, with better access to computers in wealthier households [19].

As adolescents in both country income groups seem to have wide access to TVs (but not necessarily to computers), another explanation for our main finding could be that, in low-middle-income countries, ownership of electronic devices and TVs is probably more determined by financial 


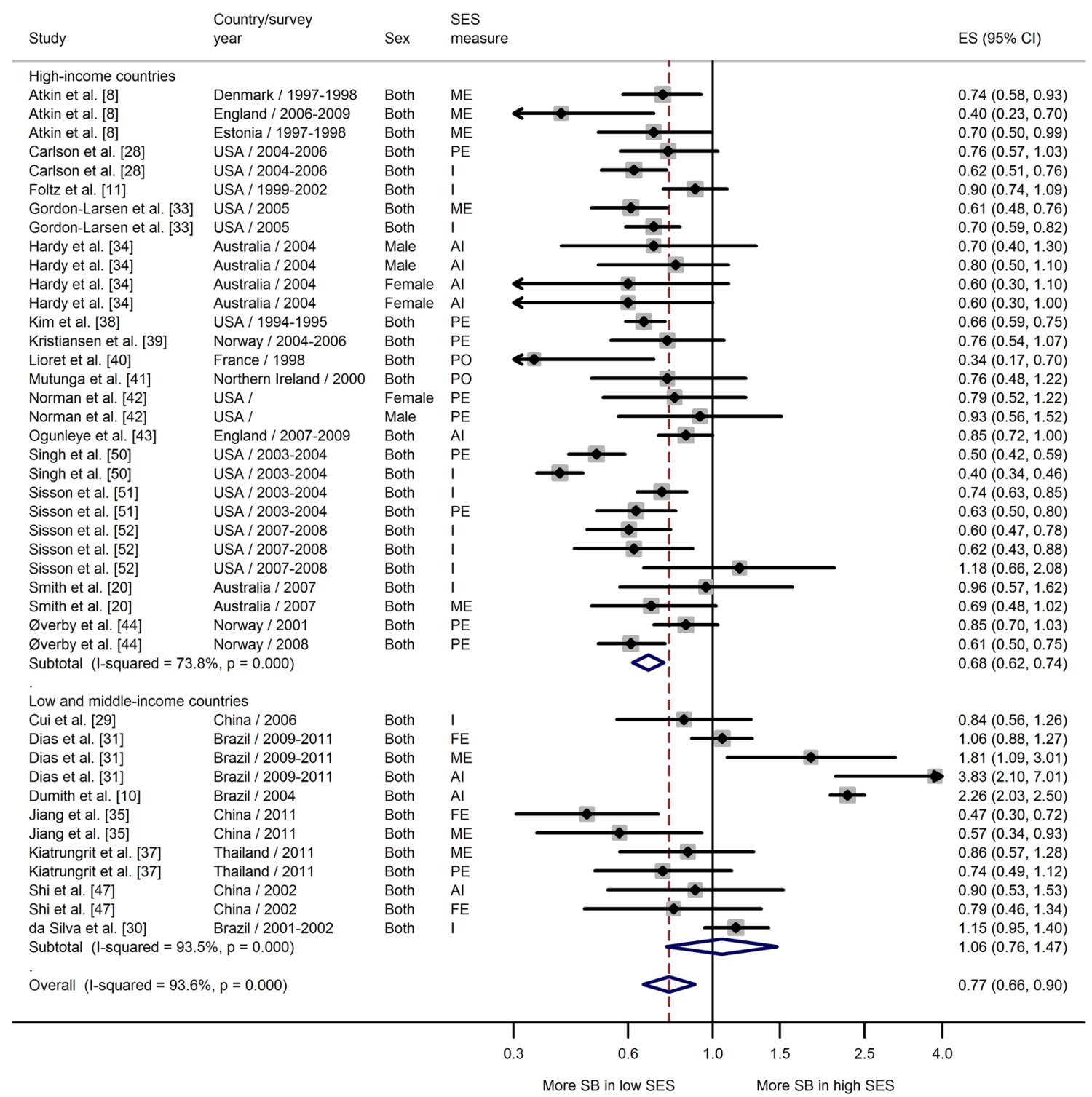

Fig. 3 Meta-analysis of the association between socioeconomic status and high screen-based time. $A I$ assets index, $C I$ confidence interval, $E S$ effect size, $F E$ paternal education, $I$ income, $M E$ maternal education, $P E$ parental education, $P O$ parental occupation, $S B$ sedentary behavior, SES socioeconomic status

One challenge in this study was the high level of variation in the sedentary behavior measures. We originally intended to develop a separate analysis for each sedentary behavior domain, but the small number of estimates for some domains, and the combinations of domains included in different measures of sedentary behavior, made this impossible. For example, only one study from a high-income country (Spain) [23] and two from low-middle-income countries (China [24] and Palestine [25]) provided estimates of study time (see ESM Fig. S1), and these were combined with video game and computer time (see ESM Fig. S2). The three broad categories of sedentary behavior used here-TV time, screen time (including TV, computer, 


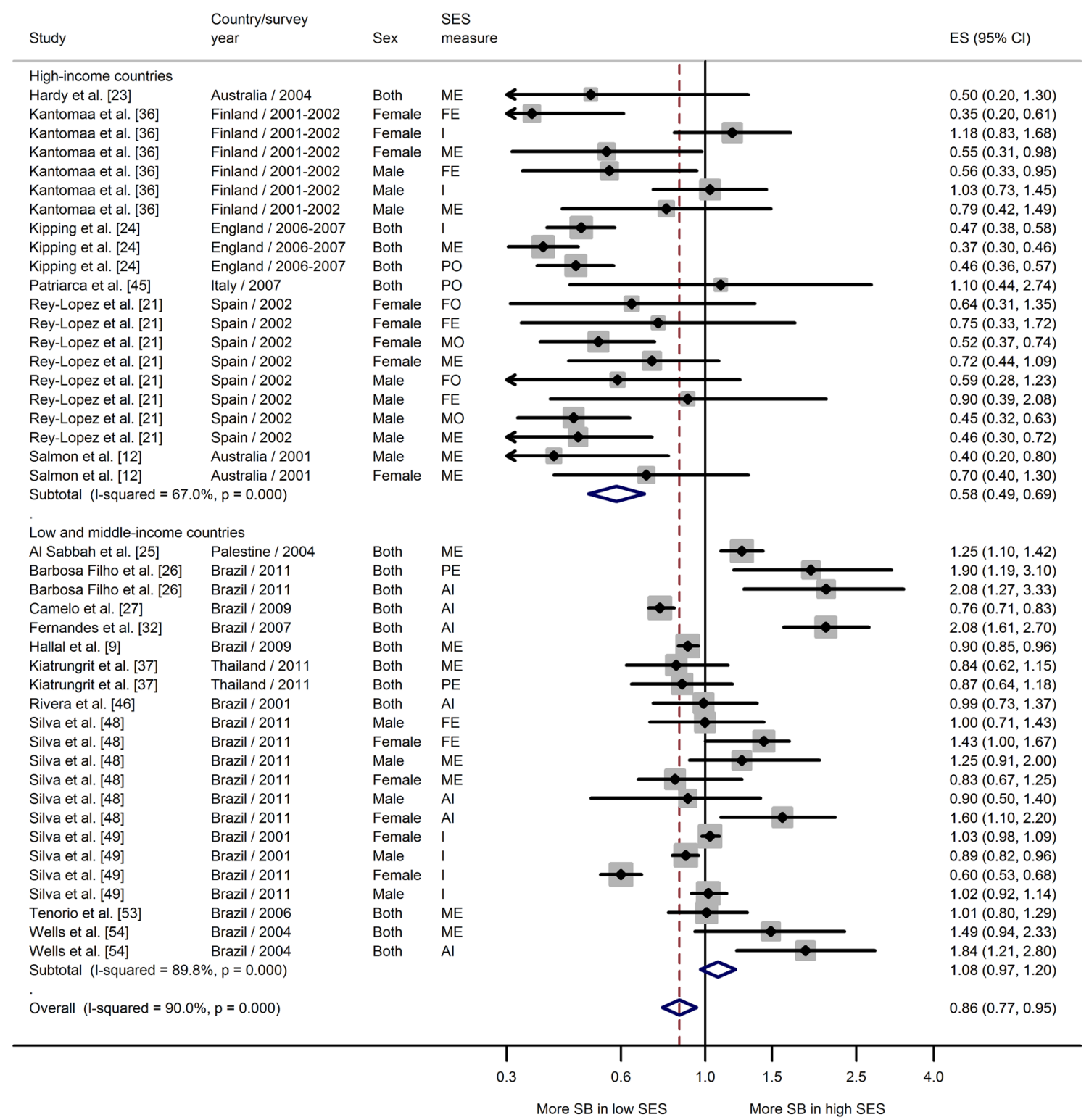

Fig. 4 Meta-analysis of the association between SES and high television viewing time. $A I$ assets index, $C I$ confidence interval, $E S$ effect size, $F E$ paternal education, $F O$ paternal occupation, $I$ income,

and game time) and "other" screen time (i.e., not including TV)—showed different patterns in the SES association, which overall seemed to reflect the socioeconomic factors relating to access, either through availability of devices, or through parental control of behaviors $[13,15]$.

A second challenge for this meta-analysis was that the definitions of "high" sedentary behavior varied across studies. Although guidelines from the American Academy of Paediatrics suggest that adolescents should not spend more than $2 \mathrm{~h}$ per day in screen-based activities [26], several different cut-points were used in the original studies. However, more than half the estimates of high sedentary behavior were based on the $2 \mathrm{~h}$ /day limit, and
$M E$ Maternal education, $M O$ maternal occupation, $P E$ parental education, $P O$ parental occupation, $S B$ sedentary behavior, $S E S$ socioeconomic status

different cut-points did not represent an important source of heterogeneity in the results. Our sensitivity analyses found the results were virtually the same when only those studies that used the $2 \mathrm{~h} /$ day cut-point were included. Furthermore, we chose not to include objective measures of sedentary behavior. Although we located studies of the association between SES and objectively measured sedentary behavior, cut-points used to define "sedentary" varied, and none of the studies provided a breakdown of time spent in different domains or a definition of "high" sedentary behavior, making it impossible to harmonize the data from objective and subjective measures. Another potential limitation was that we included separate estimates 


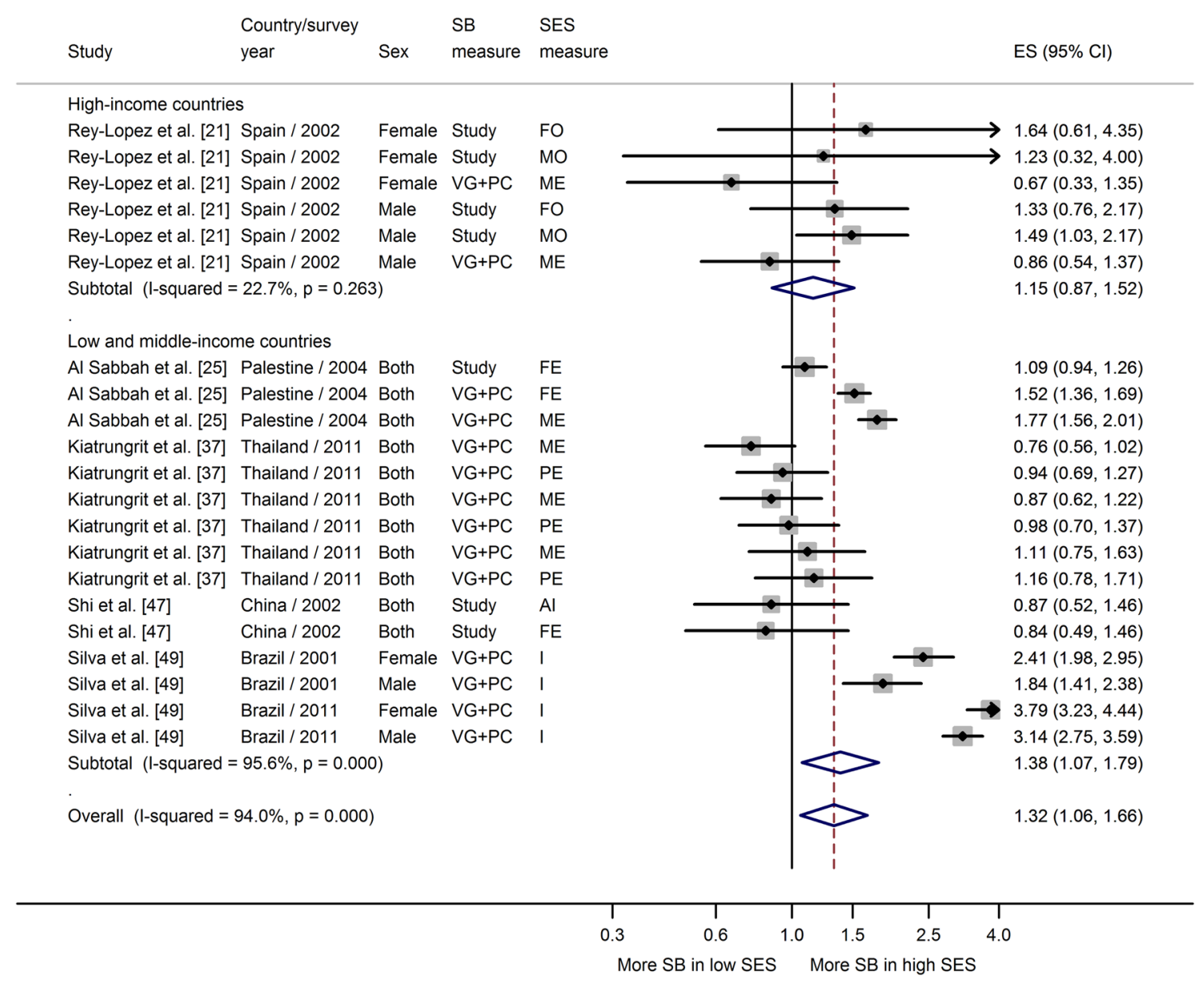

Fig. 5 Meta-analysis of the association between socioeconomic status and other sedentary behavior domains (computer, video game, study time, but not including TV time). $A I$ assets index, $C I$ confidence interval, $E S$ effect size, $F E$ paternal education, $F O$ paternal

from studies that used more than one SES indicator. This may have introduced bias and a "narrowing" of the pooled estimates. However, our sensitivity analyses showed similar results when only one estimate from each study was included.

A third challenge was that the included studies defined the different SES variables with various numbers of categories, making it difficult to pool results and potentially leading to issues of misclassification. To minimize this, we included only the extreme groups reported in each study. Sensitivity analyses showed the results were unchanged when three categories were used. However, when four or more SES categories were included, we found stronger effect measures, mainly among low-middle-income countries.

As may be expected when attempts are made to combine the results of studies that used diverse methods of data collection and varying definitions of both SES and sedentary behaviors, there was marked heterogeneity in the findings. However, a strength of our study was that we occupation, $I$ income, $M E$ maternal education, $M O$ maternal occupation, $P C$ computer, $P E$ parental education, $S B$ sedentary behavior, $S E S$ socioeconomic status, $V G$ video game

attempted to explain this heterogeneity by conducting a series of meta-regression analyses with subgroups. High heterogeneity in the first analyses led us to investigate the variation in the association between SES and sedentary behavior in adolescents from countries with different income levels, and in different sedentary behavior domains, and using different measures of SES.

This study makes an important contribution to our understanding of associations between SES and sedentary behavior in adolescents, because most previous studies have only reported results based on the presence or absence of an association, with significance indicated by $p$ values, without reporting the magnitude of the association. For example, one study whose results were included in this meta-analysis reported no "significant" associations $(p<0.05)$, but showed a strong OR, limited by a small sample size [27]. Another reported statistically "significant" findings based on very small differences in sedentary time $(<5 \%$ between the lowest and highest SES groups), but with very large samples $(>60,000)$ [9]. 
The main limitation of this study is that interpretation of these findings, especially of the overall pooled estimates, is hampered to some degree by heterogeneity and other sources of potential bias. However, the absence of publication bias, the consistency of our results identified through sensitivity analyses, and use of a more conservative random-effects model for analysis enhance the confidence we have in our conclusions. We also conducted subgroup analyses to investigate whether results differed when education was measured using paternal, maternal, or parental education; no important differences were found. In England, Kipping et al. [28] investigated associations between SES, measured by social class, maternal education, and family income. They found that, after mutual adjustment for other SES variables, family income and maternal education were both inversely associated with TV viewing time [28].

A second limitation is that, with studies from only 15 different countries, the results cannot be extrapolated worldwide. Most of the studies from low-middle-income countries came from Brazil. However, significant differences exist in the cultural, social, and economic contexts of Brazil and China, which were grouped together for the purposes of this analysis because the World Bank classifies both as middle-income countries. These differences might affect the association between SES and sedentary behavior among adolescents. In addition, although our meta-analysis included peer-reviewed publications written in English, Spanish, and Portuguese, four studies in other languages (e.g., Arabic) had to be excluded because we could not translate them.

In terms of future research, data from prospective studies that focus on determinants rather than correlates will be useful. Objective measures of sedentary behaviors, with pattern recognition to identify domains, will also advance this field. However, a need remains for studies from low- and middle-income countries other than Brazil if future interventions are to address sedentary behaviors in socially and cultural relevant contexts.

\section{Conclusion}

The findings of this review show that the relationships between SES and high sedentary behavior differ between high- and low-middle-income countries and vary by domain of sedentary behavior, and, to a lesser extent, by measure of individual SES. These complex associations between environmental, cultural, social, and individual factors and sedentary behaviors can inform the development of both local and population-based strategies that will support adolescents to choose activity over sedentariness whenever there is a possibility of choice. Our findings suggest that different approaches may be required when developing intervention strategies for reducing sedentary behavior in adolescents in different parts of the world.

\section{Compliance with Ethical Standards}

Funding Gregore Mielke was funded by a scholarship from the Coordinator for the Improvement of Higher Education Personnel (CAPES), Brazil. This work was supported by a partnership between CAPES, Federal University of Pelotas, Brazil, and the School of Human Movement and Nutrition Sciences, University of Queensland, Australia, that enabled international collaborative work to be undertaken.

Conflict of interest Gregore Mielke, Wendy Brown, Bruno Nunes, Inacio Silva, and Pedro Hallal have no conflicts of interest relevant to the content of this review.

Open Access This article is distributed under the terms of the Creative Commons Attribution 4.0 International License (http:// creativecommons.org/licenses/by/4.0/), which permits unrestricted use, distribution, and reproduction in any medium, provided you give appropriate credit to the original author(s) and the source, provide a link to the Creative Commons license, and indicate if changes were made.

\section{References}

1. Brown WJ, Bauman AE, Owen N. Stand up, sit down, keep moving: turning circles in physical activity research? Br J Sports Med. 2009;43(2):86-8.

2. Network Sedentary Behaviour Research. Standardized use of the terms "sedentary" and "sedentary behaviours". Appl Physiol Nutr Metab. 2012;37:540-2.

3. Owen N, Leslie E, Salmon J, et al. Environmental determinants of physical activity and sedentary behavior. Exerc Sport Sci Rev. 2000;28(4):153-8.

4. Van Der Horst K, Paw MJAC, Twisk JW, et al. A brief review on correlates of physical activity and sedentariness in youth. Med Sci Sports Exerc. 2007;39(8):1241-50.

5. Pate RR, Mitchell JA, Byun W, et al. Sedentary behaviour in youth. Br J Sports Med. 2011;45(11):906-13.

6. Gorely T, Marshall SJ, Biddle SJ. Couch kids: correlates of television viewing among youth. Int $J$ Behav Med. 2004;11(3): 152-63.

7. Muthuri SK, Wachira LJ, Leblanc AG, et al. Temporal trends and correlates of physical activity, sedentary behaviour, and physical fitness among school-aged children in Sub-Saharan Africa: a systematic review. Int $\mathrm{J}$ Environ Res Public Health. 2014;11(3):3327-59.

8. Atkin AJ, Sharp SJ, Corder K, et al. Prevalence and correlates of screen time in youth: an international perspective. Am J Prev Med. 2014;47(6):803-7.

9. Hallal PC, Knuth AG, Cruz DK, et al. Physical activity practice among Brazilian adolescents. Cien Saude Colet. 2010;15(Suppl 2):3035-42.

10. Dumith SC, Hallal PC, Menezes AM, et al. Sedentary behavior in adolescents: the 11-year follow-up of the 1993 Pelotas (Brazil) birth cohort study. Cad Saude Publica. 2010;26(10):1928-36.

11. Foltz JL, Cook SR, Szilagyi PG, et al. US adolescent nutrition, exercise, and screen time baseline levels prior to national recommendations. Clin Pediatr. 2011;50(5):424-33. 
12. Salmon J, Timperio A, Telford A, et al. Association of family environment with children's television viewing and with low level of physical activity. Obes Res. 2005;13(11):1939-51.

13. Braveman PA, Cubbin C, Egerter S, et al. Socioeconomic status in health research: one size does not fit all. JAMA. 2005;294(22):2879-88.

14. Galobardes B, Shaw M, Lawlor DA, et al. Indicators of socioeconomic position (part 2). J Epidemiol Community Health. 2006;60(2):95-101.

15. Fernandez-Alvira JM, Te Velde SJ, Singh A, et al. Parental modeling, education and children's sports and TV time: the ENERGY-project. Prev Med. 2015;70:96-101.

16. Hesketh K, Ball K, Crawford D, et al. Mediators of the relationship between maternal education and children's TV viewing. Am J Prev Med. 2007;33(1):41-7.

17. WHO, World Health Organization. Young People's Health-a Challenge for Society. Report of a WHO Study Group on Young People and Health for All. Technical Report Series 731. Geneva: WHO, 1986.

18. Moher D, Liberati A, Tetzlaff J, et al. Preferred reporting items for systematic reviews and meta-analyses: the PRISMA statement. BMJ. 2009;339:b2535.

19. Brazilian Institute of Geography and Statistics. Resultados gerais da amostra do Censo Demografico 2010. Rio de Janeiro: Brazilian Institute of Geography and Statistics; 2012.

20. Barbosa Filho VC, de Campos W, Bozza R, et al. The prevalence and correlates of behavioral risk factors for cardiovascular health among Southern Brazil adolescents: a cross-sectional study. BMC Pediatrics. 2012;12:130.

21. Silva DA, Tremblay MS, Goncalves EC, et al. Television time among Brazilian adolescents: correlated factors are different between boys and girls. Sci World J. 2014;2014:794539.

22. Smith BJ, Grunseit A, Hardy LL, et al. Parental influences on child physical activity and screen viewing time: a population based study. BMC Public Health. 2010;10:593.

23. Rey-Lopez JP, Tomas C, Vicente-Rodriguez G, et al. Sedentary behaviours and socio-economic status in Spanish adolescents: the AVENA study. Eur J Public Health. 2011;21(2):151-7.

24. Shi Z, Lien N, Kumar BN, et al. Physical activity and associated socio-demographic factors among school adolescents in Jiangsu Province, China. Prev Med. 2006;43(3):218-21.

25. Al Sabbah H, Vereecken C, Kolsteren P, et al. Food habits and physical activity patterns among Palestinian adolescents: findings from the national study of Palestinian schoolchildren (HBSCWBG2004). Public Health Nutr. 2007;10(7):739-46.

26. American Academy of Pediatrics. Committee on Public Education. American Academy of pediatrics: children, adolescents, and television. Pediatrics. 2001;107(2):423-6.

27. Hardy LL, Baur LA, Garnett SP, et al. Family and home correlates of television viewing in 12-13 year old adolescents: the Nepean Study. Int J Behav Nutr Phys Act. 2006;3:24.

28. Kipping RR, Smith M, Heron J, et al. Multiple risk behaviour in adolescence and socio-economic status: findings from a UK birth cohort. Eur J Public Health. 2015;25(1):44-9.

29. Camelo LV, de Castro Rodrigues JF, Giatti L, et al. Sedentary leisure time and food consumption among Brazilian adolescents: the Brazilian National School-Based Adolescent Health Survey (PeNSE), 2009. Cad Saude Publica. 2012;28(11):2155-62.

30. Carlson SA, Fulton JE, Lee SM, et al. Influence of limit-setting and participation in physical activity on youth screen time. Pediatrics. 2010;126(1):e89-96.

31. Cui Z, Hardy LL, Dibley MJ, et al. Temporal trends and recent correlates in sedentary behaviours in Chinese children. Int $\mathbf{J}$ Behav Nutr Phys Act. 2011;8:93.
32. da Silva KS, Nahas MV, Peres KG, et al. Factors associated with physical activity, sedentary behavior, and participation in physical education among high school students in Santa Catarina State, Brazil. Cad Saude Publica. 2009;25(10):2187-200.

33. Dias PJ, Domingos IP, Ferreira MG, et al. Prevalence and factors associated with sedentary behavior in adolescents. Rev Saude Publica. 2014;48(2):266-74.

34. Fernandes RA, Junior IF, Cardoso JR, et al. Association between regular participation in sports and leisure time behaviors in Brazilian adolescents: a cross-sectional study. BMC Public Health. 2008;8:329.

35. Gordon-Larsen P, McMurray RG, Popkin BM. Determinants of adolescent physical activity and inactivity patterns. Pediatrics. 2000;105(6):E83.

36. Hardy LL, Dobbins TA, Denney-Wilson EA, et al. Descriptive epidemiology of small screen recreation among Australian adolescents. J Paediatr Child Health. 2006;42(11):709-14.

37. Jiang XX, Hardy LL, Ding D, et al. Recreational screen-time among Chinese adolescents: a cross-sectional study. J Epidemiol. 2014;24(5):397-403.

38. Kantomaa MT, Tammelin TH, Näyhä S, et al. Adolescents' physical activity in relation to family income and parents' education. Prev Med. 2007;44(5):410-5.

39. Kiatrungrit K, Hongsanguansri S. Cross-sectional study of use of electronic media by secondary school students in Bangkok, Thailand. Shanghai Arch Psychiatry. 2014;26(4):216-26.

40. Kim J, Liu J, Colabianchi N, et al. The effect of perceived and structural neighborhood conditions on adolescents' physical activity and sedentary behaviors. Arch Pediatr Adolesc Med. 2010;164(10):935-42.

41. Kristiansen H, Juliusson PB, Eide GE, et al. TV viewing and obesity among Norwegian children: the importance of parental education. Acta Paediatr. 2013;102(2):199-205.

42. Lioret S, Maire B, Volatier JL, et al. Child overweight in France and its relationship with physical activity, sedentary behaviour and socioeconomic status. Eur J Clin Nutr. 2007;61(4):509-16.

43. Mutunga M, Gallagher AM, Boreham C, et al. Socioeconomic differences in risk factors for obesity in adolescents in Northern Ireland. Int J Pediatr Obes. 2006;1(2):114-9.

44. Norman GJ, Schmid BA, Sallis JF, et al. Psychosocial and environmental correlates of adolescent sedentary behaviors. Pediatrics. 2005;116(4):908-16.

45. Ogunleye AA, Voss C, Sandercock GR. Prevalence of high screen time in English youth: association with deprivation and physical activity. J Public Health (Oxf). 2012;34(1):46-53.

46. Øverby NC, Klepp K-I, Bere E. Changes in screen time activity in Norwegian children from 2001 to 2008: two cross sectional studies. BMC Public Health. 2013;13(1):1-7.

47. Patriarca A, Di Giuseppe G, Albano L, et al. Use of television, videogames, and computer among children and adolescents in Italy. BMC Public Health. 2009;9:1-10.

48. Rivera IR, Silva MA, Silva RD, et al. Physical inactivity, TVwatching hours and body composition in children and adolescents. Arq Bras Cardiol. 2010;95(2):159-65.

49. Silva KS, da Silva Lopes A, Dumith SC, et al. Changes in television viewing and computers/videogames use among high school students in Southern Brazil between 2001 and 2011. Int J Public Health. 2014;59(1):77-86.

50. Singh GK, Yu SM, Siahpush M, et al. High levels of physical inactivity and sedentary behaviors among US immigrant children and adolescents. Arch Pediatr Adolesc Med. 2008;162(8):756-63.

51. Sisson SB, Broyles ST, Baker BL, et al. Television, reading, and computer time: correlates of school-day leisure-time sedentary 
behavior and relationship with overweight in children in the U.S. J Phys Act Health. 2011;8(Suppl 2):S188-97.

52. Sisson SB, Broyles ST. Social-ecological correlates of excessive TV viewing: difference by race and sex. J Phys Act Health. 2012;9(3):449-55.

53. Tenorio MC, Barros MV, Tassitano RM, et al. Physical activity and sedentary behavior among adolescent high school students. Rev Bras Epidemiol. 2010;13(1):105-17.
54. Wells JC, Hallal PC, Reichert FF, et al. Sleep patterns and television viewing in relation to obesity and blood pressure: evidence from an adolescent Brazilian birth cohort. Int J Obes (Lond). 2008;32(7):1042-9. 